\title{
UNIQUENESS OF THE DYER-LASHOF OPERATIONS
}

\author{
JAMES E. MCCLURE
}

\begin{abstract}
It is well known that the Steenrod operations are uniquely determined by certain of their properties, namely stability, the Cartan formula, and the unstable property. In this note we give a similar characterization of Dyer-Lashof operations.
\end{abstract}

The Dyer-Lashof operations $Q^{i}$ are natural homomorphisms in the $\bmod p$ homology of $E_{\infty}$ spaces which have the following properties (see [2] and [1, I §1]; we remind the reader that $E_{\infty}$ spaces need not be connected).

(A) If $p=2, Q^{i}$ has degree $i$ and $Q^{i} x=0$ (respectively, $Q^{i} x=x^{2}$ ) if the degree of $x$ is greater than $i$ (respectively, equal to $i$ ). If $p$ is odd, $Q^{i}$ has degree $2 i(p-1)$ and $Q^{i} x=0\left(Q^{i} x=x^{p}\right)$ if the degree of $x$ is greater than $2 i$ (equal to $2 i$ ).

(B) The external Cartan formula holds:

$$
Q^{i}(x \times y)=\sum_{j \geqslant 0} Q^{j} x \times Q^{i-j} y .
$$

(C) $Q^{i} \sigma=\sigma Q^{i}$, where $\sigma: \tilde{H}_{n} \Omega X \rightarrow H_{n+1} X$ is the homology suspension.

Our main result is the following.

THEOREM. Let $R^{i}, i \geqslant 0$, be any sequence of natural homomorphisms in the homology of $E_{\infty}$ spaces satisfying (A), (B) and (C). Then $R^{i}=Q^{i}$.

This is analogous to the uniqueness theorem for Steenrod operations given in [5, Chapter VIII] and $[3, \S 10]$, but the proof will be quite different.

The proof will consist of several lemmas. To simplify the notation we begin with the case $p=2$, so that $H_{*}$ denotes mod 2 homology. The odd primary case goes through with some minor changes which will be noted at the end.

Let $E_{j} X$ denote $E \Sigma_{j} \times_{\Sigma_{j}} X^{j}$, where $E \Sigma_{j}$ is a contractible free $\Sigma_{j}$-space and the symmetric group operators on $X^{j}$ by permuting the factors. Let $i: X \times X \rightarrow E_{2} X$ be the inclusion, and let

$$
d_{j}: E_{j}(X \times Y) \rightarrow E_{j} X \times E_{j} Y
$$

be the map taking $\left(e, x_{1}, y_{1}, \ldots, x_{j}, y_{j}\right)$ to $\left(e, x_{1}, \ldots, x_{j}, e, y_{1}, \ldots, y_{j}\right)$. Recall that if $x$ is an element of $H_{n} X$ then $Q^{i} x$ is defined to be $\left(\xi_{2}\right)_{*}\left(e_{i-n} \otimes x^{2}\right)$, where the map $\xi_{2}: E_{2} X \rightarrow X$ is part of the $E_{\infty}$ structure for $X$. We can give a similar factorization for $R^{i}$ by using the construction $C X$ given in $[4, \S 2]$. The space $C\left(X^{+}\right)$is

Received by the editors April 17, 1984.

1980 Mathematics Subject Classification (1985 Revision). Primary 55S12.

(C1987 American Mathematical Society $0002-9939 / 87 \$ 1.00+\$ .25$ per page 
homeomorphic to $\left(\amalg_{j \geqslant 1} E_{j} X\right)^{+}$, and we write $\pi_{j}: C\left(X^{+}\right) \rightarrow E_{j} X$ for the projection and $\lambda: X \rightarrow X^{+}$for the inclusion. For each $j \geqslant 1$ and each (not necessarily $E_{\infty}$ ) space $X$ we let $\bar{R}_{j}^{i}$ be the composite

$$
H_{n} X \stackrel{\lambda_{*}}{\rightarrow} H_{n}\left(X^{+}\right) \stackrel{\eta_{*}}{\rightarrow} H_{n} C\left(X^{+}\right) \stackrel{R^{i}}{\rightarrow} H_{n+i} C\left(X^{+}\right) \stackrel{\pi_{j}}{\rightarrow} H_{n+i} E_{j} X ;
$$

note that if $R^{i}$ is equal to $Q^{i}$ we must have $\bar{R}_{j}^{i}=0$ for $j \neq 2$ and $\bar{R}_{2}^{i} x=e_{i-n} \otimes x^{2}$ for $x \in H_{n} X$.

Lemma 1. If $x \in H_{n} X$ then

$$
\bar{R}_{j}^{n} x= \begin{cases}i_{*}(x \times x) & \text { if } j=2, \\ 0 & \text { otherwise. }\end{cases}
$$

Proof. The diagram

$$
\begin{array}{ccc}
X \times X & \stackrel{i}{\rightarrow} & E_{2} X \\
\downarrow(\eta \circ \lambda) \times(\eta \circ \lambda) & \bigcap^{+} \\
C\left(X^{+}\right) \times C\left(X^{+}\right) \stackrel{\rightarrow}{\rightarrow} & C\left(X^{+}\right)
\end{array}
$$

where the bottom map is the product map in $C\left(X^{+}\right)$, commutes by the definition of the product. The result follows from property (A).

Lemma 2. $\left(d_{j}\right)_{*} \bar{R}_{j}^{i}(x \times y)=\sum_{k \geqslant 0} \bar{R}_{j}^{k} x \times \bar{R}_{j}^{i-k} y$.

Proof. Let $d: C\left((X \times Y)^{+}\right) \rightarrow C\left(X^{+}\right) \times C\left(Y^{+}\right)$be induced by the projections $p_{1}$ and $p_{2}$ of $(X \times Y)^{+}$onto $X^{+}$and $Y^{+}$. Then $d$ is an $E_{\infty}$ map, and hence

$$
\begin{aligned}
d_{*} R^{i} \eta_{*} \lambda_{*}(x \times y) & =R^{i} d_{*} \eta_{*} \lambda_{*}(x \times y) \\
& =R^{i}(\eta \times \eta)_{*}\left(p_{1} \times p_{2}\right)_{*} \lambda_{*}(x \times y) \\
& =R^{i}\left(\eta_{*} \lambda_{*} x \times \eta_{*} \lambda_{*} y\right) \\
& =\sum_{k \geqslant 0} R^{k} \eta_{*} \lambda_{*} x \times R^{i-k} \eta_{*} \lambda_{*} y
\end{aligned}
$$

by property (B). The result follows since $\left(\pi_{j} \times \pi_{j}\right) \circ d=d_{j} \circ \pi_{j}$.

LeMma 3. $\bar{R}_{j}^{i}=0$ for $j \neq 2$.

Proof. Let $P$ be the one-point space and let $g: E_{j} P \rightarrow P$ be the unique map. Let $e \in H_{0} P$ be the identity element. The composite

$$
E_{j} X=E_{j}(X \times P) \stackrel{d_{j}}{\rightarrow} E_{j} X \times E_{j} P \stackrel{1 \times g}{\rightarrow} E_{j} X \times P=E_{j} X
$$

is the identity. Hence for any $x \in H_{n} X$ we have

$$
\bar{R}_{j}^{i} x=(1 \times g)_{*}\left(d_{j}\right)_{*} \bar{R}_{j}^{i}(x \times e)=\sum_{k \geqslant 0} \bar{R}_{j}^{i-k} x \times g_{*} \bar{R}_{j}^{k} e
$$

by Lemma 2. Now $g_{*} \bar{R}_{j}^{k} e=0$ for $k>0$ for dimensional reasons, and $\bar{R}_{j}^{0} e=0$ if $j \neq 2$ by Lemma 1 . The result follows. 
LEMmA 4. If $X$ is an $E_{\infty}$ space and $x \in H_{n} X$ then $R^{i} x=\left(\xi_{2}\right)_{*} \bar{R}_{2}^{i} x$.

Proof. Consider the diagram

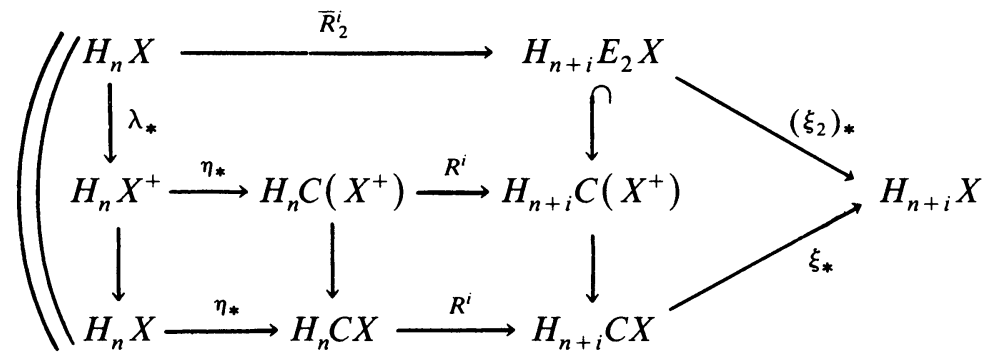

Here $\xi$ is the map given by the $E_{\infty}$ structure and the triangle commutes by definition of $\xi$ and $\xi_{2}$. The upper rectangle commutes by Lemma 3. The two lower rectangles (in which the unmarked arrows are induced by the projection $X^{+} \rightarrow X$ ) commute by naturality. Since $\xi$ is an $E_{\infty}$ map we have $\xi_{*} R^{i} \eta_{*} x=R^{i} \xi_{*} \eta_{*} x=R^{i} x$ as required.

By Lemma 4 it suffices to show $\bar{R}_{2}^{i}=\bar{Q}_{2}^{i}$ for all $i$, where $\bar{Q}_{2}^{i}$ is constructed from $Q^{i}$ in the same way that $\bar{R}_{2}^{i}$ is constructed from $R^{i}$.

As usual, we filter $C X$ by letting $F_{k} C X$ be the image of

$$
\left(\coprod_{1 \leqslant j \leqslant k} E_{j} X\right)^{+} \subset C\left(X^{+}\right) \rightarrow C X .
$$

We write $F_{k} H_{*} C X$ for the image of the map $H_{*} F_{k} C X \rightarrow H_{*} C X$; recall from the proof of [1, Theorem I.4.1] that this map is in fact a monomorphism. By Lemma 3 we see that $R^{i} \eta_{*} x$ is in $F_{2} H_{*} C X$ for any $x \in H_{n} X$.

Now let $s_{n} \in \tilde{H}_{n} S^{n}$ be the fundamental class.

LEMMA 5. $R^{i} \eta_{*} s_{n}=Q^{i} \eta_{*} s_{n}$ in $H_{n+1} C\left(S^{n}\right)$ for all $i$ and $n$.

Proof. The result is immediate from property (A) if $i \leqslant n$, so we assume $i>n$. The proof of [1, Theorem I.4.1] shows that $F_{2} H_{n+i} C\left(S^{n}\right)$ is generated by $Q^{i} \eta_{*} s_{n}$, hence $R^{i} \eta_{*} s_{n}$ is a scalar multiple of $Q^{i} \eta_{*} s_{n}$. To determine what multiple we consider the composite

$$
F_{2} H_{n+i} C S^{n} \subset H_{n+i} C S^{n} \rightarrow H_{n+i} Q S^{n}=H_{n+i} \Omega^{i-n} Q S^{i} \stackrel{\sigma^{i-n}}{\rightarrow} H_{2 i} Q S^{i} .
$$

By properties (A) and (C) this map takes both $Q^{i} \eta_{*} s_{n}$ and $R^{i} \eta_{*} s_{n}$ to $\left(\eta_{*} s_{i}\right)^{2} \neq 0$. The result follows.

Next we must consider a reduced version of $\bar{R}_{2}^{i}$. Let $D_{2} X$ denote $E Z_{2}^{+} \wedge_{Z_{2}}(X \wedge$ $X)$. The maps $i$ and $d_{2}$ pass to the quotient to give

$$
\iota: X \wedge X \rightarrow D_{2} X \text { and } \delta_{2}: D_{2}(X \wedge Y) \rightarrow D_{2} X \wedge D_{2} Y \text {. }
$$

Let $\tilde{R}^{i}$ be the composite

$$
\tilde{H}_{n} X \subset H_{n} X \stackrel{\bar{R}_{2}^{i}}{\rightarrow} H_{n+i} E_{2} X \rightarrow \tilde{H}_{n+i} D_{2} X,
$$


where the last arrow is induced by the evident quotient map $E_{2} X \rightarrow D_{2} X$. By Lemmas 1 and 2 we have

$$
\tilde{R}^{n} x=\iota_{*}(x \wedge x) \text { if } x \in \tilde{H}_{n} X
$$

and

$$
\left(\delta_{2}\right)_{*} \tilde{R}^{i}(x \wedge y)=\sum_{j \geqslant 0} \tilde{R}^{j} x \wedge \tilde{R}^{i-j} y .
$$

The space $D_{2}\left(X^{+}\right)$is homeomorphic to $\left(E_{2} X\right)^{+}$, and it is easy to see that the composite

$$
H_{n} X=\tilde{H}_{n} X^{+} \stackrel{\tilde{R}^{i}}{\rightarrow} \tilde{H}_{n+i} D_{2}\left(X^{+}\right)=H_{n+i} E_{2} X
$$

is $\bar{R}_{2}^{i}$. Thus to prove the theorem it suffices to show $\tilde{R}^{i}=\tilde{Q}^{i}$ for all $i$.

LEMMA 6. $\tilde{R}^{i} s_{n}=\tilde{Q}^{i} s_{n}$ for all nonnegative $i$ and $n$.

Proof. The quotient map $E_{2} X \rightarrow D_{2} X$ factors as

$$
E_{2} X \rightarrow F_{2} C X \rightarrow F_{2} C X / F_{1} C X \simeq D_{2} X .
$$

Since $H_{*} F_{2} C X \rightarrow H_{*} C X$ is monic, we see from Lemma 5 that $\bar{R}_{2}^{i} s_{n}$ and $\bar{Q}_{2}^{i} s_{n}$ have equal images in $H_{n+i} F_{2} C S^{n}$. Hence they have equal imges in $\tilde{H}_{n+i} D_{2} S^{n}$.

Next recall from [6] that any element $x \in \tilde{H}_{n} X$ is represented by a based map

$$
h_{x}: S^{n+m} \rightarrow K\left(Z_{2}, m\right) \wedge X
$$

for some $m$. By Lemma 6 we have

$$
\left(\delta_{2}\right)_{*} \tilde{R}^{i+m}\left(h_{x}\right)_{*} s_{n+m}=\left(\delta_{2}\right)_{*} \tilde{Q}^{i+m}\left(h_{x}\right)_{*} s_{n+m}
$$

in $\tilde{H}_{*} K\left(Z_{2}, m\right) \otimes \tilde{H}_{*} X$. We wish to compute the components of each side of (3) in $\tilde{H}_{2 m} K\left(Z_{2}, m\right) \otimes \tilde{H}_{n+i} X$. Let $\varepsilon_{m}^{*}$ be the fundamental class of $\tilde{H}^{m} K\left(Z_{2}, m\right)$ and let $\varepsilon_{m}$ be its dual. The definitions imply that the slant product $\varepsilon_{m}^{*} \backslash\left(h_{x}\right)_{*} s_{n+m}$ is $x$, hence $\left(h_{x}\right)_{*} s_{n+m}=\varepsilon_{m} \otimes x$ modulo higher degrees in $\tilde{H}_{*} K\left(Z_{2}, m\right)$. Now equation (2) gives

$$
\left(\delta_{2}\right)_{*} \tilde{R}^{i+m}\left(h_{x}\right)_{*} s_{n+m}=\tilde{R}^{m} \varepsilon_{m} \otimes \tilde{R}^{i} x
$$

modulo higher degrees in $\tilde{H}_{*} D_{2} K\left(Z_{2}, m\right)$. The same calculation holds for $\tilde{Q}^{i+m}$, so we have

$$
\tilde{R}^{m} \varepsilon_{m} \otimes \tilde{R}^{i} x=\tilde{Q}^{m} \varepsilon_{m} \otimes \tilde{Q}^{i} x .
$$

But by equation (1) we have $\tilde{R}^{m} \varepsilon_{m}=\tilde{Q}^{m} \varepsilon_{m}=\iota^{*}\left(\varepsilon_{m} \wedge \varepsilon_{m}\right)$, and the latter element is nonzero by [1, Theorem I.4.1]. Thus equation (4) implies the theorem when $p=2$.

When $p$ is odd the argument must be modified somewhat. The number 2 should be replaced by $p$ throughout and all two-fold cartesian and smash products $X \times X$ and $X \wedge X$ replaced by $p$-fold products. The operation $R^{i}$ increases degrees by $2 i(p-1)$ instead of by $i$. In addition, the following specific changes are needed. Lemma 1 should read:

If $x \in H_{2 n} X$ then

$$
\bar{R}_{j}^{n}= \begin{cases}i_{*} x^{p} & \text { if } j=p \\ 0 & \text { otherwise }\end{cases}
$$


In the proof of Lemma 5 we may assume that $2 i>n$, and the map $\sigma^{i-n}$ should be replaced by

$$
\sigma^{2 i-n}: H_{n+2 i(p-1)} \Omega^{2 i-n} Q S^{2 i} \rightarrow H_{2 p i} Q S^{2 i} .
$$

Equation (1) should be replaced by

$$
\tilde{R}^{n} x=i_{*}\left(x^{(p)}\right) \text { if } x \in \tilde{H}_{2 n} X .
$$

In the argument following Lemma 6 the map $h_{x}$ should go from $S^{n+2 m}$ to $K\left(Z_{p}, 2 m\right) \wedge X$ for some $m$. The elements $s_{n+m}$ and $\varepsilon_{m}$ in that argument should be replaced by $s_{n+2 m}$ and $\varepsilon_{2 m}$. To conclude the argument one computes the components of each side of equation (3) in the group $\tilde{H}_{2 p m} K\left(Z_{p}, 2 m\right) \otimes \tilde{H}_{n+2 i(p-1)} X$.

\section{REFERENCES}

1. F. R. Cohen, T. J. Lada and J. P. May, The homology of iterated loop spaces, Lecture Notes in Math., vol. 533, Springer-Verlag, Berlin and New York, 1976.

2. E. Dyer and R. K. Lashof, Homology of iterated loop spaces, Amer. J. Math. 84 (1962), 35-88.

3. J. P. May, A general algebraic approach to Steenrod operations, The Steenrod Algebra and its Applications (F. P. Peterson, ed.), Lecture Notes in Math., vol. 168, Springer-Verlag, Berlin and New York, 1970, pp. 153-231.

4. __ The geometry of iterated loop spaces, Lecture Notes in Math., vol. 271, Springer-Verlag, Berlin and New York, 1972.]

5. N. E. Steenrod and D. B. A. Epstein, Cohomology operations, Ann. of Math. Studies, no. 50, Princeton Univ. Press, Princeton, N. J., 1962.

6. G. W. Whitehead, Generalized homology theories, Trans. Amer. Math. Soc. 102 (1962), 227-283.

Department of Mathematics, University of Chicago, Chicago, Illinois 60637

Current address: Department of Mathematics, University of Kentucky, Lexington, Kentucky 40506 